\title{
Médiévales
}

Langues, Textes, Histoire

51 | automne 2006

L'Occident sur ses marges ( $\mathrm{VI}{ }^{\mathrm{e}}-\left.\mathrm{X}\right|^{\mathrm{e}}$ siècles)

\section{Les « miroirs aux princes » sont-ils un genre littéraire?}

Einar Már Jónsson

\section{(2) OpenEdition}

1 Journals

\section{Édition électronique}

URL : https://journals.openedition.org/medievales/1461

DOI : 10.4000/medievales.1461

ISSN : 1777-5892

Éditeur

Presses universitaires de Vincennes

\section{Édition imprimée}

Date de publication : 1 décembre 2006

Pagination : 153-166

ISBN : 978-2-84292-193-4

ISSN : 0751-2708

\section{Référence électronique}

Einar Már Jónsson, "Les « miroirs aux princes » sont-ils un genre littéraire ? », Médiévales [En ligne], 51 | automne 2006, mis en ligne le 27 mars 2009, consulté le 23 avril 2022. URL : http://

journals.openedition.org/medievales/1461; DOI : https://doi.org/10.4000/medievales.1461

Ce document a été généré automatiquement le 23 avril 2022

Tous droits réservés 


\title{
Les « miroirs aux princes » sont-ils un genre littéraire?
}

\author{
Einar Már Jónsson
}

1 Depuis fort longtemps on a pris l'habitude de désigner un certain nombre de traités politiques médiévaux dans l'Occident chrétien par le terme générique de « miroirs aux princes » (Fürstenspiegel en allemand, mirror of princes en anglais). Sur ces œuvres, il existe maintenant une littérature abondante, mais autant que nous le sachions, les nombreux auteurs qui les ont étudiées se sont surtout intéressés à leur contenu politique et aux informations qu'elles peuvent nous fournir sur l'idéologie royale au Moyen Âge. En revanche, ils ne se sont guère occupés de l'élaboration littéraire de ces "miroirs aux princes », qui est en fait très variée, et ils ne se sont pas interrogés non plus sur le titre générique et son rapport éventuel avec le contenu. Dans les plus anciennes études que nous ayons trouvées à ce sujet, un article d'Albert Werminghoff paru en $1902^{1}$, et un livre d'Ernst Booz qui date de $1913^{2}$, les auteurs utilisent le terme «miroir au prince» sans aucune définition ni explication. On remarque en particulier qu'ils ne le font pas dériver d'une expression médiévale et ne le mettent pas non plus en relation avec un titre d'ouvrage précis ou une métaphore employée par un auteur du Moyen Âge ${ }^{3}$. Dans l'étude de Booz, on sent d'ailleurs que la terminologie n'est pas encore tout à fait fixée, car à côté de Fürstenspiegel, l'auteur emploie parfois, dans le même sens, le mot Fürstenschrift et également l'expression plus longue et précise, «fürstenerzieherische Schriften (Traktaten, Werke)». Ces termes sont strictement synonymes, et on devine même qu'à cette époque Fürstenspiegel est utilisé simplement comme un terme plus imagé et frappant que Fürstenschrift, mais sans une portée plus grande.

2 Par la suite, c'est le mot «miroir aux princes » que les médiévistes ont retenu, et il figure dans les titres de plusieurs études des années 1930, notamment celle de Wilhelm Berges, Die Fürstenspiegel des hohen und späten Mittelalters (1938), qui l'a popularisé ${ }^{4}$. Ainsi, il semble bien qu'il se soit imposé comme une sorte d'évidence, mais sans jamais avoir été défini ou explicité. Pourtant, ce mot n'est pas neutre : beaucoup plus chargé de sens que par exemple Fürstenschrift, il renvoie à un symbolisme philosophique et 
théologique, et rattache également ces traités politiques à un groupe d'autres œuvres médiévales, intitulées "miroir», telles que le Speculum maius de Vincent de Beauvais. Tout cela pose en fait beaucoup de questions, et derrière elles, il y a une question fondamentale : de quelle façon peut-on dire que les « miroirs aux princes » forment un genre littéraire?

C'est sur ces questions que nous voulons nous pencher ici, mais avant d'aborder le sujet une précision s'impose. Nous n'avons aucunement la prétention d'être un spécialiste des «miroirs aux princes", et si nous nous y sommes intéressé, c'est à cause d'une recherche que nous avons faite il y a bien longtemps sur le Speculum regale, ou « Miroir royal ", une des œuvres les plus importantes de la littérature scandinave du Moyen Âge, écrite en Norvège, et en langue norvégienne malgré le titre latin, et datée de 1260 environ ${ }^{5}$. Cette œuvre se rattache clairement à la tradition littéraire et intellectuelle du Moyen Âge occidental, et pour nous la question était de savoir de quelle façon précisément.

4 À l'époque, ce problème paraissait déjà définitivement réglé. Tout le monde, que ce soit les spécialistes norvégiens ou les savants étrangers, était d'accord pour dire que le Speculum regale était un "miroir au prince " parmi d'autres. Déjà Wilhelm Berges lui avait fait une place de choix ${ }^{6}$, et la grande philologue norvégienne Anne Holtsmark avait résumé ce qui était certainement le consensus des savants à l'époque dans son article pour le Kulturhistorisk leksikon for nordisk middelalder, publié en 1964 dans le tome IX. Il est déjà symptomatique que l'article ne soit pas consacré au Speculum regale en particulier; cette œuvre norvégienne est groupée avec un traité politique suédois du XIV siècle, intitulé Um styrilsi kununga ok höfdhinga ("Sur le gouvernement des rois et des princes »), avec lequel elle n'a certainement aucune relation, le tout sous le titre générique «littérature de miroirs aux princes». Voici comment Anne Holtsmark résume ce qui était considéré comme une évidence :

Avec le mot speculum, "miroir", on a désigné depuis le haut Moyen Âge des manuels de morale. En norrois, ce mot (skuggsiá, en norrois de l'ouest) est utilisé pour la première fois dans ce sens dans la traduction du livre d'Alcuin sur les vertus et les vices: dans les livres sacrés on peut se voir soi-même comme dans un miroir, skuggsio. Dans la littérature occidentale il y avait de nombreux «miroirs" de ce genre spécialement destinés aux rois et aux fils de rois.

Cependant, ce consensus ne reposait sur rien. Aucun savant n'avait en effet entrepris d'étudier les relations précises entre le Speculum regale et les "miroirs aux princes» pour chercher s'il y avait entre les deux de véritables rapports formels, structurels et thématiques qui permettraient de situer l'œuvre norvégienne parmi les «miroirs aux princes » et éventuellement lui assigner une place précise dans ce corpus. Berges luimême ne s'était intéressé qu'à certaines idées du traité, sans l'étudier dans sa totalité et sans le situer dans une perspective globale. Du coup il nous semblait que la recherche sur le Speculum regale se trouvait dans une certaine impasse, d'où l'on ne pourrait guère sortir sans chercher du côté que les savants avaient jusqu'alors négligé. Cette recherche, qu'il était devenu urgent de faire, comportait à notre avis deux aspects différents, et par là elle sortait de la problématique particulière du Speculum regale norvégien et touchait celle des «miroirs aux princes " globalement en tant que genre littéraire ${ }^{7}$.

6 1) Il fallait d'abord chercher si ce genre reposait sur quelque chose qu'on pourrait appeler un « fondement formel », notamment des titres typiques justifiés par le recours à des métaphores précises voire à un symbolisme lié aux sujets des œuvres. Cette 
question s'impose d'emblée, car si ce que dit Anne Holtsmark est vrai, si l'on trouvait dans ces écrits la métaphore du miroir appliquée précisément à un manuel de morale ou d'éthique politique destiné à un roi ou à un prince, le problème serait en partie résolu, on aurait déjà une définition concrète du genre littéraire, ou du moins un fil conducteur pour l'explorer. Ceci est d'autant plus fondamental que le terme même de « miroirs aux princes » suppose déjà l'existence d'une symbolique de ce genre.

7 2) Il fallait ensuite analyser les œuvres classées habituellement comme « miroirs aux princes » pour voir quels rapports structurels et thématiques il y avait éventuellement entre elles. En d'autres mots, il fallait établir une sorte de typologie des «miroirs aux princes " pour pouvoir définir la place de chaque texte dans le corpus, et pour cerner, le cas échéant, l'évolution du genre, les influences des œuvres les unes sur les autres, ou les influences extérieures au corpus.

$C^{\prime}$ est dans ces deux directions que nous nous sommes engagé ${ }^{8}$. Dans cette recherche nous nous sommes limité aux œuvres antérieures aux années 1260, non seulement à cause de l'interrogation initiale, concernant l'œuvre norvégienne que l'on date en général des dernières années du roi Hákon le vieux, mort en 1263, mais aussi parce qu'il nous semblait que l'atmosphère intellectuelle avait à tel point changé dans la dernière partie du XIII ${ }^{e}$ siècle que le problème devait se poser différemment à partir de cette date. Même s'il y a quelques points de contact, notre étude s'arrête donc au seuil de ce groupe de «miroirs aux princes» capétiens sur lequel Jean-Philippe Genet s'est penché 9 .

9 Il est en fait assez facile de répondre à la première question, concernant l'idée même d'un « miroir au prince » dans le sens littéral de ce terme, mais la réponse est peut-être inattendue. Avant la fin $\mathrm{du} \mathrm{XIII}^{\mathrm{e}}$ siècle, en tout cas, on ne trouve pas trace de la "métaphore du miroir » appliquée à un manuel de morale ou d'éthique politique destiné à un roi ou un prince, et, à part deux œuvres, le Speculum regum de Godefroid de Viterbe et le Speculum regale norvégien, textes marginaux dont les titres en tout cas ne signifient pas « miroir au prince », les œuvres classées comme Fürstenspiegel ne portent jamais le titre de "speculum $»{ }^{10}$. Celles qui portent véritablement ce titre, suivi d'un qualificatif, comme par exemple le speculum virginum, le speculum caritatis ou le Speculum maius, appartiennent à un autre genre littéraire qui n'a rien à voir avec des « miroirs aux princes ». Cela appelle peut-être quelques éclaircissements.

10 Il ne semble pas que le mot "miroir " ait été utilisé au Moyen Âge comme un nom commun pour désigner des manuels de morale. Le passage d'Alcuin que cite Anne Holtsmark, et qui est tiré du De virtutibus et vitiis, n'est en aucune façon un exemple de ce sens particulier; il illustre par contre le sens bien plus technique et précis du mot. Dans ce texte, en effet, Alcuin n'est pas en train de parler d'un quelconque « manuel de morale ", il compare les écritures saintes à un "miroir ». Il s'agit là d'un topos très répandu à toutes les époques du Moyen Âge et qui comporte de nombreuses variations : le « miroir » peut désigner par métaphore les livres sacrés eux-mêmes, la Bible, des vies de saints etc., la Révélation divine que contiennent ces livres sacrés, ou encore autre chose qui reflète par d'autres intermédiaires la Révélation divine. Au début, un livre écrit par un auteur ne pouvait guère être qualifié de "miroir", sauf s'il contenait véritablement un «reflet» de la Révélation, tel que le Speculum Augustini qui est un florilège biblique ${ }^{11}$.

11 Ce n'est qu'au début du XII siècle que nous trouvons véritablement, avec le Speculum ecclesiae d'Honorius Augustodunensis et le Speculum virginum anonyme, un «livre- 
miroir », ou « Buchspiegel » comme disent les Allemands, c'est-à-dire un livre écrit par un auteur, auquel celui-ci a donné le titre de "miroir", suivi d'un qualificatif qui en précise le sens. Les titres de ces premiers « miroirs » sont dérivés des topoi antérieurs et ils sont expliqués par des symbolismes élaborés, notamment dans le Speculum virginum, qui les rattachent à l'idée du « miroir de la Révélation » ${ }^{12}$.

À partir de ces deux œuvres, le genre littéraire des "miroirs" se développe et se répand dans l'Occident chrétien aux $\mathrm{XII}^{\mathrm{e}}$ et $\mathrm{XIII}^{\mathrm{e}}$ siècles. Ce n'est pas notre propos ici de retracer cette longue évolution, que j'espère, deo iuvante, étudier ailleurs, mais nous voudrions souligner deux aspects :

13 1) Les «miroirs" des $\mathrm{XII}^{\mathrm{e}}$ et $\mathrm{XIII}^{\mathrm{e}}$ siècles, c'est-à-dire les œuvres qui portaient véritablement les titres de miroirs, sont très variés, et certains d'entre eux pourraient être qualifiés de "manuels de morale » ou de comportement. Mais ces œuvres-là sont toujours destinées à des religieux: il existe bel et bien un Speculum virginum, un Speculum monachorum et un Speculum novitii, mais nous ne trouvons nulle part un "Speculum militis », un "Speculum mercatorum » ou encore, dans ce sens précis, un "Speculum regum ». Pour des raisons qui sont peut-être liées au sens religieux de la métaphore, il ne peut y avoir, à cette époque, des «miroirs " destinés à des classes particulières de laïcs.

2) Nous avons dit plus haut que la métaphore du miroir pouvait désigner divers reflets de la Révélation divine, non seulement les Écritures et les textes écrits, mais aussi d'autres formes de vision indirecte. C'est ainsi que l'on trouve dans certains " miroirs " de cette période la métaphore du «miroir de la créature ", c'est-à-dire de la créature comme miroir du Créateur. On peut alors penser que, par une métaphore analogue, on pourrait avoir l'idée d'un ordre historico-politique voulu par Dieu lui-même comme un miroir du Créateur, et donc des specula politiques. Effectivement, on trouve cette idée, mais dans des œuvres assez marginales dans le corpus des «miroirs» de l'époque. La plus importante qui la contienne est le Sachsenspiegel, qui est, comme on le sait, une somme juridique de première importance, mais pas un «miroir au prince ». Une autre œuvre, plus marginale encore, est le Speculum regum de Godefroid de Viterbe. Les références au symbolisme du miroir que l'on trouve dans le prologue indiquent que pour l'auteur ce livre est à la fois un miroir qui montre au destinataire (le roi et futur empereur Henri VI) l'histoire des anciens rois et le miroir que les anciens rois présentent au destinataire pour lui permettre de connaître sa propre situation dans l'histoire. Mais le titre n'a aucunement le sens de "miroir au prince», tel que les savants modernes l'ont défini, et l'œuvre n'est pas un "manuel de morale » ou d'éthique politique mais un résumé d'histoire.

On peut donc dire que si le genre littéraire des "miroirs" aux XII et XIII siècles déborde d'une façon marginale sur le domaine politique et touche, si l'on peut dire, le genre des Fürstenspiegel, la métaphore concrète $d u$ « miroir au prince » est absente.

Ce qui est en réalité le plus frappant, ce n'est pas cette absence de la seule métaphore $\mathrm{du}$ « miroir au prince » dans le genre littéraire auquel on a sans doute abusivement donné ce nom, mais l'absence complète d'une métaphore quelconque, d'un symbolisme ou de titres typiques qui auraient pu fonder le genre littéraire sur une base symbolique et le délimiter ainsi en établissant des frontières dans le champ littéraire de l'époque. En regardant les œuvres que les savants ont classées comme des " miroirs aux princes » on constate en effet que les titres sont presque toujours purement "factuels", se 
référant concrètement au sujet de l'œuvre ou au rapport entre l'auteur et le roi auquel il s'adresse, et d'une extrême diversité.

17 En ce qui concerne la référence concrète au sujet, on trouve de nombreux titres comme De regis persona et regio ministerio (de Hincmar de Reims, vers 873), Liber de rectoribus christianis (de Sedulius Scotus, vers 855), De regimine principum (de Hélinand de Froidmont, vers 1200), De principis instructione (de Giraud de Barri, vers 1217), Liber de regimine civitatum (de Jean de Viterbe, vers 1228), Eruditio regum et principum (de Guibert de Tournai, 1259), L'enseignement des princes (de Robert de Blois, vers le milieu du XIII ${ }^{\mathrm{e}}$ siècle), et De morali principis instructione de Vincent de Beauvais (vers 1260). Le terme générique qui conviendrait le mieux à ces écrits serait effectivement, en se fondant sur les titres, Fürstenerzieherische Schriften (" manuel d'éducation princière »).

Les titres qui se réfèrent au rapport entre l'auteur et le roi sont bien moins nombreux, mais on relève : La seconde élégie pour le roi Pépin d'Aquitaine d'Ermold le noir (vers 828) et Admonitio Jonae episcopi ad Pippinum de Jonas d'Orléans (vers 831, connu aujourd'hui sous le titre De institutione regia), auxquels on pourrait peut-être ajouter les épitres Ad proceres regni et Ad episcopos regni de Hincmar de Reims (882). Les titres du premier groupe peuvent d'ailleurs aussi être complétés par le nom du destinataire. Ainsi le titre complet de l'œuvre majeure de Hincmar est: De regis persona et regio ministerio ad Carolum regem.

19 Certains « miroirs aux princes » ont un caractère historique qui est mis en évidence par les titres: le Carolinus de Gilles de Paris (vers 1200) et la Philippide de Guillaume le Breton (1214-1224). Vu le contenu de l'œuvre et le rapport entre le titre et ce contenu, on devrait sans doute situer ici le Speculum regum, déjà cité, de Godefroid de Viterbe (1180-1183).

20 Finalement on trouve deux titres qui ont une référence métaphorique et symbolique, mais pas la même : Via regia de Smaragde de Saint-Mihiel (800-814) et Morale somnium pharaonis, sive de regia disciplina de Jean de Limoges (1255-1260).

21 De cette diversité des titres on ne peut tirer qu'une seule conclusion: les écrivains médiévaux, en tout cas jusqu'à la deuxième moitié du xiII ${ }^{\mathrm{e}}$ siècle, n'avaient pas de nom pour désigner ce que les savants modernes ont appelé des "miroirs aux princes", et même pas de terme technique (tel que tractatus, summa, etc.) pour indiquer la nature de l'œuvre. Dans ces conditions on pourrait même se demander si ce qu'on a appelé le "genre littéraire des miroirs aux princes » existe réellement, si on n'a pas groupé ensemble, sous un terme générique contestable, ou même faux, des écrits qui sont en réalité tout à fait disparates. Jean-Philippe Genet, qui part de la liste de Wilhelm Berges, a tout a fait raison de dire qu'il n'y a pas d'homogénéité dans cette collection ${ }^{13}$. Pour y voir clair, il convient d'abord de demander si l'on peut trouver une définition claire qui englobe une partie aussi grande que possible des œuvres que les savants ont étudiées sous la rubrique "miroirs aux princes». Nous pensons que cela est possible, et nous reprenons une définition que nous avons déjà proposée ailleurs ${ }^{14}$ :

Un « miroir au prince » est un traité écrit pour un prince - et en général dédié à lui

- qui a pour objet principal de décrire le prince idéal, son comportement, son rôle

et sa situation au monde.

22 Cette définition a le premier avantage d'établir une frontière nette entre les écrits qu'on peut véritablement définir comme des «miroirs aux princes » et toutes sortes de traités politiques, d'œuvres de piété personnelle etc., qui ne s'adressent pas plus à des rois qu'à d'autres personnes, ou qui ne s'adressent à des rois qu'en tant que chrétiens 
ordinaires. Du coup certaines œuvres qui ont souvent été associées aux «miroirs aux princes » se trouvent rejetées hors du corpus: par exemple le De duodecim abusivis saeculi du Pseudo-Cyprien, de nombreuses epistulae exhortatoriae de l'époque carolingienne ${ }^{15}$ et La somme le roi du frère Laurent, qui sont des œuvres de piété personnelle, ou encore le Policraticus de Jean de Salisbury, un traité de grande envergure qui compare la « vie de cour » et la « vie de philosophie » et qui est adressé à Thomas Beckett ${ }^{16}$. Mais si l'on veut avoir des notions claires sur un genre littéraire, il faut établir des frontières aussi précises que possible ${ }^{17}$.

Or, les «miroirs aux princes" antérieurs aux années soixante du XIII ${ }^{\mathrm{e}}$ siècle, qui correspondent véritablement à cette définition et qui sont une vingtaine environ, sont cependant très disparates. Il s'avère néanmoins possible de les classer d'après leur thématique et leur structure, c'est-à-dire d'en établir une sorte de typologie. On peut ainsi définir quatre classes :

1) Les « miroirs aux princes » les plus simples ne font qu'énumérer les diverses vertus que le prince idéal doit posséder et, éventuellement, les vices qu'il doit éviter, et les discuter l'une après l'autre : ce sont les œuvres que les historiens allemands appellent Tugendkatalog. Dans cette première classe, on peut situer la Via regia de Smaragde de Saint-Mihiel, L'enseignement des princes de Robert de Blois, et la première partie du De principis instructione de Giraud de Barri, mais on trouve de tels " catalogues de vertus » dans beaucoup d'autres œuvres plus élaborées. Ces catalogues peuvent se présenter de diverses façons: dans la Via regia, il s'agit d'une simple énumération et les divers chapitres sont intitulés "De timore», "De sapientia», "De prudentia», "De simplicitate », etc., mais dans L'enseignement des princes, les armes et les vêtements du chevalier sont présentés comme les symboles des vertus que le roi doit posséder et qui sont énumérées, ainsi que les vices qu'il doit éviter. Mais le principe est toujours le même.

2) Une deuxième catégorie est formée par les œuvres qui décrivent le prince idéal par le biais d'un ou de plusieurs rois historiques proposés comme modèles pour leurs successeurs ou pour d'autres rois. Cette classe est en fait étroitement liée à la précédente, et la Seconde élégie d'Ermold le Noir pour Pépin d'Aquitaine illustre bien la relation entre les deux catégories. Ce texte, qui est assez court, commence par un «catalogue de vertus» copié sur la Via regia de Smaragde. Puis l'auteur continue en donnant des exemples de rois vertueux : d'abord David et Salomon, mais ces exemples bibliques amènent des exemples plus récents, Pépin de Herstal, Charles Martel, et suit finalement une généalogie des Carolingiens avec une exhortation au roi Pépin d'Aquitaine de suivre l'exemple de ses illustres ancêtres. Une sorte d'inversion de ce thème apparaît dans le De principis instructione de Giraud de Barri. Là, le « catalogue des vertus » est suivi d'un exemple récent (Henri II d'Angleterre), mais c'est pour montrer le triste sort d'un roi qui ne possède pas les vertus précédemment énumérées. Dans une autre œuvre, le Carolinus de Gilles de Paris, l'auteur utilise les quatre vertus cardinales comme principe de composition pour exposer l'histoire de Charlemagne qu'il propose comme modèle. Deux écrits, qu'on peut ranger ici, le Speculum regum de Godefroid de Viterbe et la Philippide de Guillaume le Breton, sont plus proches d'œuvres historiques pures et ne contiennent pas de « catalogue des vertus ».

3) La troisième classe des « miroirs aux princes » contient les œuvres qui ne se limitent pas à énumérer les vertus du prince idéal, mais qui le décrivent plus systématiquement dans l'exercice de ses fonctions. Leur sujet est donc double: quelles sont les 
caractéristiques du roi juste et comment peut-il faire régner la justice. Cette définition n'est pourtant qu'une indication, car les textes qu'on peut classer ici sont en fait extrêmement disparates. On peut citer en premier le De regis persona et regio ministerio de Hincmar de Reims, qui se borne surtout à étudier le roi idéal comme chef de guerre et comme juge, et le Liber de rectoribus christianis de Sedulius Scotus, qui présente une étude d'ensemble sur le «bonus princeps » (en le comparant avec le "rex impius ») et sur son attitude envers tous ceux qui l'entourent. L'Eruditio regum et principum de Guibert de Tournai est également une étude systématique qui part de la discipline du roi envers lui-même pour examiner ensuite comment il peut corriger ses sujets. Mais on pourrait aussi classer ici des œuvres assez divergentes, telle que le Morale somnium pharaonis où Jean de Limoges imagine un curieux échange de lettres entre Joseph et le Pharaon: le rêve de ce dernier devient ainsi un prétexte pour parler du roi idéal, critiquer les défauts du " Pharaon » et de la cour et exhorter à des réformes.

4) La quatrième et dernière classe des "miroirs aux princes " ressemble beaucoup à celle que je viens de décrire, de telle sorte que les frontières sont parfois difficiles à établir, mais ce qui change ici, c'est que les sujets sont traités plus à fond et l'horizon est plus étendu. Au lieu de décrire simplement le roi idéal et son action, les auteurs le mettent dans un contexte élargi et étudient systématiquement la question du pouvoir et le rapport entre le regnum et le sacerdotium. Parfois l'accent se déplace également, et au lieu de parler du prince idéal, les auteurs traitent de la royauté en général et de sa fonction. Dans cette classe on peut notamment citer l'Admonitio Jonae episcopi ad Pippinum de Jonas d'Orléans (connu sous le titre de De institutione regia, qui correspond en fait au sujet mais qui a été donné par un éditeur moderne), et les deux lettres écrites par Hincmar de Reims en 882, Ad proceres regni et Ad episcopos regni. L'œuvre du juriste Jean de Viterbe, le De regimine principum, qui était destiné non pas à un roi mais à un podestà italien, est encore plus systématique, contenant toutes les définitions (celles de regimen, civitas, potestas, du pouvoir du pape et de l'empereur etc.) dans le cadre général d'une description de sa fonction depuis le début jusqu'à la fin. Dans cette quatrième classe on peut également situer le De morali principis instructione, de Vincent de Beauvais, et le De eruditione principum, de Guillaume Peyraut plus tard faussement attribué à Thomas d'Aquin ${ }^{18}$.

Entre ces quatre classes de "miroirs aux princes » il y a des rapports étroits: on pourrait facilement parler d'une progression constante depuis la première jusqu'à la dernière, pas seulement parce que chaque classe ajoute un aspect ou un thème nouveau à la précédente (dont elle reprend souvent les thèmes), mais aussi parce qu'avec chaque nouvelle classe la réflexion sur le roi idéal s'approfondit. Du simple catalogue des vertus, on passe à une étude systématique de la royauté dans tout son contexte. Mais pourtant cette progression n'est qu'une vue de l'esprit, car il n'y a aucun rapport temporel, ou de succession, entre ces différentes classes : elles coexistent depuis le début jusqu'à la fin de la période qui nous concerne ici. Parmi les six « miroirs aux princes » carolingiens, on trouve déjà des exemples de ces quatre classes, et on les trouve également parmi les œuvres postérieures, des $\mathrm{XII}^{\mathrm{e}}$ et $\mathrm{XIII}$ e siècles. Il n'est donc pas étonnant que H. H. Anton, qui n'étudie que les œuvres carolingiennes, puisse conclure en disant que ces «miroirs aux princes" n'ont aucune unité formelle: "den karolingischen Fürstenspiegel in formaler Hinsicht gab es nicht ${ }^{19}$. Il se contente ensuite d'établir une liste de l'ensemble des sujets discutés dans les textes en question: la seule chose qui constitue l'unité du genre, c'est le fait que chacun prend comme sujet de discussion un ou plusieurs thèmes (Themenkreisen) de cette liste ${ }^{20}$. Bien que la liste 
de H. H. Anton soit faite à partir des œuvres carolingiennes, elle contient les sujets des quatre classes, et on pourrait la considérer dans son ensemble comme une sorte de table de matière idéale de la quatrième classe, la plus étendue puisqu'elle est susceptible d'englober aussi les sujets des autres. Légèrement transformée et adaptée à la classification que nous venons de proposer la liste se présente ainsi :

1) le rapport entre le pouvoir du roi et le pouvoir de l'Église, la situation du roi dans l'ordre universel voulu par Dieu, la définition du mot rex et l'opposition rex /tyrannus ;

2) Virtutes regiae, le roi idéal, la morale du roi dans sa vie privée et dans la politique, la récompense du roi après la mort (et son châtiment le cas échéant);

31 3) exemples de rois (Saul, David, Salomon, les ancêtres du roi pour lequel l'œuvre est écrite);

32 4) la fonction du roi, son rôle de protecteur des faibles et de modèle pour ses sujets. Ses méthodes pour guider ses sujets vers le bon chemin et maintenir la vertu dans son royaume. liste, les vertus, mais les autres ajoutent d'autres thèmes jusqu'à ce qu'ils soient tous traités dans les œuvres de la quatrième classe.

fait que tous ces thèmes coexistent depuis l'époque carolingienne et que leur " accumulation", si l'on peut dire, ne représente pas une progression temporelle, nous oblige à poser de nouveau la question de l'unité de ce genre varié. Les œuvres se sontelles influencées mutuellement, y a-t-il un modèle commun? Curieusement, dans les deux cas la réponse est négative. Les auteurs de «miroirs aux princes » de l'époque carolingienne connaissaient souvent les écrits des autres auteurs qui écrivaient sur le même sujet, mais ne les utilisaient guère ${ }^{21}$, les œuvres carolingiennes n'ont pas eu d'influence par la suite - on n'a même pas d'indication qu'on les ait lues plus tard ${ }^{22}$ - et les écrivains des $\mathrm{XII}^{\mathrm{e}}$ et $\mathrm{XIII}^{\mathrm{e}}$ siècles ont apparemment composé isolément les uns des autres ${ }^{23}$. Les textes qui ont inspiré ces auteurs d'une façon générale, tels que le $D e$ civitate Dei de saint Augustin pour les deux périodes, ou le De duodecim abusivis saeculi du Pseudo-Cyprien pour l'époque carolingienne, sont des sources générales pour les idées et non pas pour le choix des thèmes ou pour la structure des œuvres. En cherchant les sources plus précises pour les œuvres individuelles, on découvre qu'elles sont extérieures au genre, et, en général, différentes dans chaque cas.

On pourrait même aller plus loin et rattacher les différentes classes des «miroirs aux princes » à des genres différents de la littérature occidentale. La première classe est en effet très proche des œuvres de morale en général, ou des œuvres de piété destinées à d'autres professions : on peut le constater à propos de la Via regia de Smaragde où l'auteur cite abondamment son propre Diadema monachorum ${ }^{24}$. De même, la deuxième classe tend à se confondre avec l'historiographie : il n'y a pas grand-chose qui sépare le Speculum regum ou la Philippide d'une œuvre historique ordinaire. Finalement, les deux dernières classes sont proches du traité de philosophie politique : il est par exemple assez probable que si Vincent de Beauvais avait pu achever selon le plan initial l'œuvre commencée avec le De eruditione puerorum nobilium et le De morali principis institutione, qu'il appelle d'ailleurs une fois " opus universale » ${ }^{25}$, celle-ci ne serait pas devenue un « miroir au prince » mais une sorte d'encyclopédie politique.

36 Faudrait-il alors conclure de cette absence de rapports littéraires que le genre des «miroirs aux princes» n'existe pas et qu'il faille disperser ailleurs, dans d'autres 
genres littéraires, les œuvres qu'on a groupées sous cette rubrique ? Cela dépend, bien entendu, de la définition qu'on adopte du concept "genre littéraire ». Si on le définit à partir de critères formels, et en même temps programmatiques, qui annoncent un choix particulier de sujets et une attitude particulière envers les sujets, tels que les titres typiques "somme", "sentences", "miroir » etc., alors il est clair que les « miroirs aux princes » ne forment pas un genre littéraire. Si on le définit, d'un point de vue plutôt historique, comme un groupe d'œuvres littéraires dont les auteurs s'inspiraient des mêmes modèles, s'influençaient mutuellement et suivaient les mêmes règles, on est obligé de dire que les "miroirs aux princes " ne forment pas un genre littéraire non plus. Si, enfin, on adopte un critère purement matériel et qu'on définisse un genre littéraire comme un groupe d'écrits qui parlent du même sujet, telles que les œuvres historiques, alors les «miroirs aux princes» ne peuvent guère être définies comme un genre littéraire homogène. En tout état de cause, les Fürstenspiegel ne sont donc pas un genre littéraire au même titre, par exemple, que les œuvres intitulées Speculum ou Summa.

Tout cela ne veut cependant pas dire que l'on doive refuser toute unité à ce corpus qu'on a désigné par le nom "miroirs aux princes». Ces œuvres forment incontestablement un groupe. Apparemment, elles sont situées dans un lieu incertain du champ littéraire médiéval où se rejoignent les œuvres de morale, l'historiographie et les traités politiques, et elles oscillent parfois entre ces trois genres en les mêlant. Mais, d'une façon paradoxale, leur unité surgit à travers cet enchevêtrement. Dans ces œuvres, le thème du «prince idéal» devient en effet le centre de tout un ensemble thématique, où les thèmes moraux, historiques et politiques venus de divers horizons se combinent les uns avec les autres et forment différentes structures, comme la classification esquissée plus haut le montre.

Les raisons de cet enchevêtrement et de l'unité qui en résulte sont cependant extralittéraires : les œuvres sont écrites indépendamment les unes des autres mais dans des situations analogues, ou, plus exactement, dans une relation précise avec le prince et reflètent ainsi l'ensemble des idées qui lui sont attachées. Lorsqu'on ne fait que très peu ou pas de différence entre la morale publique et le comportement privé, lorsqu'on pense que le rôle du roi est d'être vertueux et de faire régner la vertu - car seul le rex iustus est légitime - et lorsqu'on considère l'histoire, et surtout l'histoire biblique, comme magistra uitae pour des hommes d'action, il est clair que la personne du roi réunit en elle-même tous les aspects qui forment les "miroirs aux princes" dès le départ.

[Cet article est fondé sur une communication faite dans le cadre d'une conférence sur les « miroirs aux princes » arabes organisée par Houari Touati, le 21 mai 1999.]

\section{NOTES}

1. A. WERMINGHOFF, « Die Fürstenspiegel der Karolingerzeit », Historische Zeitschrift, 89, 1902, p. 193-214. 
2. E. Booz, Die Fürstenspiegel des Mittelalters bis zur Scholastik, Fribourg en Br., 1913. Nous tenons à remercier très vivement Monique Paulmier de l'Atelier Vincent de Beauvais (ARTEM) de l'Université de Nancy II pour nous avoir aidé à dénicher ces deux textes peu connus, et pour l'aide inestimable qu'elle nous a fournie pour cette étude. 3. A. Werminghoff remarque la métaphore du miroir dans le prologue du De institutione regia de Jonas d'Orléans, et l'interprète ainsi (« Die Fürstenspiegel der Karolingerzeit », loc. cit., p. 200) : « Pippins Character lässt ihn hoffen, Gehör zu finden, auf dass der König aus dem Traktat wie aus einem Spiegel das erkenne, was er zu thun, was er zu meiden habe ». Il n'essaie cependant pas d'établir une relation entre le titre générique Fürstenspiegel et ce passage de l'œuvre de Jonas, qui n'a sans doute pas le sens qu'il lui attribue (cf. notre ouvrage Le Miroir, naissance d'un genre littéraire, Paris, 1995, p. 150-151).

4. Parmi les autres études de cette époque, on peut citer J. RÖDER, Das Fürstenbild in den mittelalterlichen Fürstenspiegeln auffranzösischem Boden, Emstetten, 1933, et W. KLEINEKE, Englische Fürstenspiegel vom Policraticus bis zum Basilikon Doron König Jakobs I, Halle, 1937.

5. Nous en avons publié une traduction française : Le Miroir royal, Nyon, 1997.

6. Wilhelm BERGES, Die Fürstenspiegel des hohen und späten Mittelalters, Stuttgart, 1938, rééd. 1952, p. 159-185.

7. De son côté Sverre Bagge a fait une comparaison entre le Miroir royal et les « miroirs aux princes » occidentaux, mais d'un autre point de vue, car il s'est surtout intéressé au contenu, les idées politiques ; cf. S. BAGGE, The Political Thought of the King's Mirror, Odense, 1987.

8. Nous avons publié les résultats de cette étude, en ce qui concerne le Miroir royal norvégien, dans notre article « La situation du Speculum regale dans la littérature occidentale ", Études germaniques, 42, 1987, p. 391-408.

9. Cf. Jean-Philippe GENET, Four English Political Tracts of the Late Middle Ages, Londres, 1977, p. ix-xix, et ID., « Saint Louis : Le roi politique », Médiévales, 34, printemps 1998, p. 27-30. Pour les « miroirs aux princes » capétiens en général, voir Jacques KRYNEN, L'Empire du roi. Idées et croyances politiques en France (XIII $-X V^{e}$ siècle), Paris, 1993,

p. 167-239.

10. C'est seulement au XIV ${ }^{\mathrm{e}}$ siècle que l'on trouve des œuvres intitulées Le Mireoirs as princes (1327, de Watriquet de Couvin), Speculum regis Edmundi II (1337-1349, attribué à Simon Islip), et Speculum morale regium (1384, de Robert de Gervais), mais à cette époque le titre « miroir » ne semble plus avoir le même sens qu'auparavant. De plus, ces œuvres paraissent marginales dans la tradition des « miroirs aux princes ». Selon le Lexikon des Mittelalters, t. IV, col. 1046, il est même douteux qu'on puisse inclure le Speculum regis dans ce genre.

11. Édition par F. WeIHRICH, CSEL 12. Voir E. DeKKERS, Clavis Patrum Latinorum, $3^{e}$ éd., Turnhout, 1995, 0272. Cf. aussi notre ouvrage : Le Miroir, naissance d'un genre littéraire, op. cit., p. 121-122.

12. Sur toute cette problématique, $c f$. notre ouvrage (voir note précédente).

13. J.-Ph. GENET, Four English Political Tracts, op. cit., p. x.

14. Cf. E. M. JonsSON, « La situation du Speculum regale dans la littérature occidentale », loc. cit., p. 394.

15. Cf. H. H. ANTON, Fürstenspiegel und Herrscherethos in der Karolingerzeit, Bonn, 1968.

16. Berges inclut le Policraticus parmi les « miroirs aux princes » qu'il étudie, op. cit., p. 131-143. 
17. Notre point de vue est donc différent de celui de Jacques LE Goff (cf. Saint Louis, Paris, 1996, p. 402-431) qui s'intéresse avant tout aux idées. Mais quelle que soit la définition, il est probable que la « frontière » du genre des « miroirs aux princes » reste toujours floue. Que faut-il faire des œuvres dont une partie seulement correspond à la définition ? Le terme allemand « fürstenspiegelachtig » pourrait encore être utile...

18. Sur ces deux œuvres, voir J. KRYNEN, op. cit., p. 173-179.

19. H. H. ANTON, Fürstenspiegel und Herrscherethos, op. cit., p. 89, n. 64.

20. Ibid.

21. Jonas d'Orléans connaît apparemment l'œuvre de Smaragde, mais ne l'utilise guère (cf. H. H. ANTON, op. cit., p. 216). Sedulius Scotus n'utilise ni l'œuvre de Smaragde ni celle de Jonas (cf. ibid., p. 263, n. 582). Hincmar semble avoir connu l'œuvre de Jonas mais il se soucie plus de compléter que de copier (cf. ibid., p. 222 et 286).

22. Cf. BERGES, op. cit., p. 3.

23. Le De principis instructione de Giraud de Barri et le Speculum regum de Godefroid de Viterbe ne semblent avoir eu aucune influence (cf. BERGES, op. cit., p. 294). La Philippide de Guillaume le Breton, qui semble avoir été écrite comme réponse au Carolinus de Gilles de Paris, a eu une certaine influence par la suite mais sur l'historiographie, pas sur d'autres « miroirs aux princes » (cf. H. F. DELABORDE, Euvres de Rigord et de Guillaume le Breton I, Paris, 1885, p. lxxvii). De même, le Liber de regimine principum de Jean de Viterbe a influencé Li livres dou tresor de Brunetto Latini, mais pas les Fürstenspiegel postérieurs (cf. BERGES, op. cit, p. 299). Nous laissons de côté les œuvres capétiennes, postérieures à l'époque qui nous concerne ici.

24. Cf. H. H. ANTON, op. cit., p. 133 sq.

25. Dans le prologue du De eruditione filiorum nobilium (cf. l'édition d'Arpad STEINER, p. 3, et BERGES, op. cit., p. 305, voir aussi l'édition de Robert SCHNEIDER, Turnhout, 1995 [CCCM 137]).

\section{AUTEUR}

\section{EINAR MÁR JÓNSSON}

Université Paris IV-Sorbonne, UFR d'Études germaniques, Département d'études nordiques, Centre universitaire Malesherbes, 108 boulevard Malesherbes, F-75010 Paris. 